\title{
HOSPITALIZAÇÕES POR FRATURA DO FÊMUR ENTRE IDOSOS EM RORAIMA, BRASIL
}

\section{COMUNICAÇÃO}

BORGHETTI, Gina ${ }^{1}$

BORGHETTI, Gina. Hospitalizações por fratura do fêmur entre idosos em Roraima, Brasil. Revista Científica Multidisciplinar Núcleo do Conhecimento. Ano. 07, Ed. 01, Vol. 02, pp. 111-119. Janeiro de 2022. ISSN: 2448-0959, Link de acesso: https://www.nucleodoconhecimento.com.br/saude/fratura-do-femur, DOI: 10.32749/nucleodoconhecimento.com.br/saude/fratura-do-femur

\section{RESUMO}

Introdução: As fraturas do fêmur em idosos aumentam o risco de morbimortalidade, sendo geralmente necessária à sua hospitalização. Devido a relevância do tema em saúde pública e a falta de informações sobre a situação no Estado no período entre 2015 a 2019, surgiu o seguinte questionamento: De acordo com a faixa etária e gênero, qual o perfil das internações hospitalares por fratura do fêmur entre os idosos em Roraima neste período? Objetivo: Descrever as internações hospitalares por fratura do fêmur entre os idosos, no período entre 2015 a 2019 no Estado de Roraima, Brasil. Método: Trata-se de um estudo descritivo, de abordagem quantitativa, utilizando dados do Sistema de Informações Hospitalares do Sistema Único de Saúde no período de 2015 a 2019. Para as análises de frequência foram utilizados os códigos referentes a fratura do fêmur de acordo a $10^{\underline{a}}$ revisão da Classificação Internacional de Doenças, faixa etária, sexo e óbitos hospitalares. Resultados: Observou-se que gênero feminino foi o mais prevalente nas internações por fratura do fêmur $(56,4 \%)$. Estratificando por faixa etária, 80 anos e mais representou $38,7 \%$ das internações, sendo na sua maioria mulheres. Este mesmo perfil foi observado quando analisadas as internações por fratura do fêmur com

\footnotetext{
${ }^{1}$ Doutorado em Biologia Celular e Molecular, Mestrado em Biologia Celular e Molecular, Graduação em Fisioterapia. ORCID: https://orcid.org/0000-0002-2922-9090.
} 
localização proximal. Das internações com desfecho de óbito por fratura do fêmur houve o predomínio entre as mulheres com idade de 80 anos e mais $(66,7 \%)$. Considerações finais: Neste estudo, observou-se que as internações e os óbitos hospitalares por fratura do fêmur foram mais prevalentes nas mulheres octogenárias. Estratégias em saúde, como a prevenção de quedas, deve ser direcionada para a população idosa, em especial às mulheres, que são as mais acometidas por este agravo.

Palavras-chave: Fraturas do Fêmur, Hospitalização, Idoso, Saúde Pública.

\section{INTRODUÇÃO}

A transição demográfica ocorre no Brasil simultaneamente com o crescente e progressivo envelhecimento da população, afetando os serviços de saúde e as condições socioeconômicas da sociedade (TRAVASSO et al., 2020). Fatores relacionados com o envelhecimento em associação com a fragilidade, osteoporose e a presença de comorbidades aumentam a ocorrência das fraturas entre os idosos (PETERLE et al., 2019; WU et al., 2019). Estudo realizado no Brasil no ano de 2013, apontou uma prevalência de três doenças simultâneas na população com idade de 60 anos ou mais. Outros fatores como perda da função muscular e baixo desempenho físico também são indicativos de eventos desfavoráveis nesta população (PETERLE et al., 2019).

Grande parte das fraturas do fêmur em idosos são secundárias as quedas acidentais, sendo o tratamento cirúrgico o procedimento mais utilizado (BORTOLON et al., 2011; ARMOND et al., 2014). No Brasil, a prevalência de pelo menos uma queda em 2019 variou entre $25,1 \%$ a 37,07\% (SANTOS et al., 2021). Entretanto, este evento não deve ser visto como parte do envelhecimento natural (PORTO et al., 2019). As quedas poderiam ser prevenidas com intervenções através de exercícios físicos orientados, identificação precoce dos fatores de risco e o tratamento da osteoporose, principalmente nas mulheres pós menopausa (BEER e GILES, 2005). 
Pesquisas indicam que o aumento das fraturas femorais está relacionado com o avanço da faixa etária e o gênero (BORTOLON et al., 2011). Estas fraturas podem ocorrem em diferentes áreas anatômicas do fêmur, como a região proximal, diáfise e a região distal (EDELMUTH et al., 2018). Estudos indicam que as fraturas localizadas no fêmur proximal, principalmente decorrentes da osteoporose, exibem maior risco de morbimortalidade e de fraturas recorrentes (CHEN et al., 2018; CARMO e GOMES, 2008).

As fraturas do fêmur aumentam o risco de mortalidade entre os idosos, principalmente se associado a fatores como, faixa etária, comorbidades, gênero e a presença de deficiência cognitiva (PETERLE et al., 2019; NETO et al., 2011). Foi demonstrado que após um ano do tratamento cirúrgico, cerca de $50 \%$ dos idosos apresentaram dificuldades nas atividades diárias e na deambulação (MACEDO et al., 2019). Geralmente apresentam redução de autonomia e perda da qualidade de vida (MACEDO et al., 2019; NASCIMENTO, 2019). Adicionalmente, podem causar impactos psicossociais e gerar custos financeiros adicionais para o sistema de saúde e familiar, pois estes idosos geralmente requerem maior tempo de internação e reabilitação (SANTOS et al., 2021; OLIVEIRA e BORBA, 2017).

As fraturas do fêmur em idosos aumentam o risco de morbimortalidade, sendo geralmente necessária à sua hospitalização. Informações sobre as internações hospitalares podem auxiliar no conhecimento desta situação em saúde e nas estratégias de prevenção para esta população. Devido a relevância do tema em saúde pública e a falta de informações sobre a situação no Estado no período entre 2015 a 2019, surgiu o seguinte questionamento: De acordo a faixa etária e gênero, qual o perfil das internações hospitalares por fratura do fêmur entre os idosos em Roraima neste período?

Diante do contexto apresentado, este estudo tem como objetivo descrever as internações hospitalares por fratura do fêmur entre os idosos, no período entre 2015 a 2019 no Estado de Roraima, Brasil. 


\section{MÉTODO}

Trata-se de um estudo descritivo, de abordagem quantitativa. A base de dados foi coletada a partir do Sistema de Informações Hospitalares do Sistema Único de Saúde (SIH/SUS), que tem origem nas Autorizações de Internações Hospitalares (AlH'S) do Departamento de Informática do SUS (DATASUS). A base de dados foi selecionada por Unidade da Federação (Roraima) e ano/mês de processamento (período entre janeiro de 2015 a dezembro de 2019). Para descrever as internações hospitalares por fratura do fêmur foram selecionadas as seguintes variáveis: Capítulos da Classificação Internacional de Doenças (CID-10), Óbitos (com óbito, sem óbito), Faixa etária (60 a 69 anos, 70 a 79 anos e 80 anos e mais) e Sexo (feminino, masculino). Foram analisadas as AlH'S registradas na base de dados SIH/SUS como Fratura do Fêmur, de acordo a $10^{\text {a }}$ revisão da Classificação Internacional de Doenças (CID-10) contidas no capítulo XIX, Lesões, envenenamento e outras consequências de causas externas. Em uma análise foram incluídos todos os códigos relativos à fratura do fêmur (S72), sem especificar a localização anatômica. $\mathrm{Na}$ segunda análise, foram incluídos somente códigos específicos $(720,721,722)$ referentes à fratura do fêmur proximal. A proporção de óbitos hospitalares entre os idosos foi analisada através da razão entre o número de idosos internados por fratura do fêmur com desfecho de óbito pelo número de idosos internados por fatura do fêmur. Os arquivos foram transferidos para o programa de tabulação TAB para Windows (TabWin) para a análise dos dados. Foi utilizado estatística descritiva, sendo calculadas as frequências absolutas (n) e relativas (\%) do número de internações para cada variável.

Como os dados coletados são de domínio público, disponibilizados em meio eletrônico e sem a identificação dos participantes da pesquisa, este estudo não necessitou de apreciação e aprovação pelo Comitê de Ética em Pesquisa.

\section{RESULTADOS}

Em Roraima, a proporção de idosos internados no período entre 2015 a 2019 foi de $11,4 \%$ (23,676). Deste total, os idosos internados por fratura do fêmur 
representaram 2,4\% (578). O gênero feminino foi o mais prevalente nas internações por fratura do fêmur $(56,4 \%)$. Estratificando por faixa etária, 80 anos e mais representou $38,7 \%$ das internações, sendo mais prevalente em mulheres $(69,6 \%)$ (Tabela-1). Quando analisado as internações, especificando a localização anatômica em fratura do fêmur proximal, as mulheres representaram a maioria $(60,6 \%)$. Estratificando por faixa etária, 80 anos e mais, representou $44,7 \%$, sendo a maioria mulheres $(70,3 \%)$ (Tabela-2). A ocorrência das internações por fratura do fêmur entre os idosos com desfecho de óbito representou 5,2\% (30), sendo na sua maioria mulheres com idade igual ou superior a 80 anos $(66,7 \%)$

Tabela - 1 Internações por fratura do fêmur (sem diferenciar a localização anatômica) por faixa etária e sexo. Roraima, Brasil, 2015 a 2019

\begin{tabular}{|l|l|l|l|l|l|l|}
\hline & \multicolumn{2}{|l|}{ Feminino } & \multicolumn{2}{l|}{ Masculino } & \multicolumn{2}{l|}{ Total } \\
\cline { 2 - 7 } & $\mathrm{n}$ & $\%$ & $\mathrm{n}$ & $\%$ & $\mathrm{n}$ & $\%$ \\
\hline $\mathbf{6 0}$ a 79 anos & 90 & 45,9 & 106 & 54,0 & 196 & 100 \\
\hline $\mathbf{7 0}$ a 79 anos & 80 & 50,6 & 78 & 49,3 & 158 & 100,0 \\
\hline $\begin{array}{l}\mathbf{8 0} \text { anos e } \\
\text { mais }\end{array}$ & 156 & 69,9 & 68 & 30,3 & 224 & 99,9 \\
\hline Total & $\mathbf{3 2 6}$ & $\mathbf{5 6 , 4}$ & $\mathbf{2 5 2}$ & $\mathbf{4 3 , 6}$ & $\mathbf{5 7 8}$ & $\mathbf{1 0 0 , 0}$ \\
\hline
\end{tabular}

Fonte: SIH/SUS, DATASUS.

Tabela - 2 Internações por fratura do fêmur (localização anatômica de fêmur proximal) por faixa etária e sexo. Roraima, Brasil, 2015 a 2019.

\begin{tabular}{|l|l|l|l|l|l|l|}
\hline & \multicolumn{2}{l|}{ Feminino } & \multicolumn{2}{l|}{ Masculino } & \multicolumn{2}{l|}{ Total } \\
\cline { 2 - 6 } & $\mathrm{n}$ & $\%$ & $\mathrm{n}$ & $\%$ & $\mathrm{n}$ & $\%$ \\
\hline $\mathbf{6 0}$ a 79 anos & 33 & 54,1 & 28 & 45,9 & 61 & 100,0 \\
\hline $\mathbf{7 0}$ a 79 anos & 33 & 51,5 & 31 & 48,4 & 64 & 100,0 \\
\hline $\begin{array}{l}\text { 80 anos e } \\
\text { mais }\end{array}$ & 71 & 70,3 & 30 & 29,7 & 101 & 100,0 \\
\hline
\end{tabular}




\begin{tabular}{l|l|l|l|l|l|l|} 
Total & 137 & 60,6 & 89 & 39,3 & 226 & 100,0
\end{tabular}

Fonte: SIH/SUS, DATASUS.

\section{DISCUSSÃO}

Segundo registros do SIH/SUS, no período de 2015 a 2019, o Capítulo XIX, Lesões, envenenamento e outras consequências de causas externas, ocupou a sexta posição entre as causas das internações hospitalares entre os idosos em Roraima, Brasil. Deste capítulo, a fratura do fêmur representou a principal causa de hospitalização. Este tipo de fratura é uma das lesões traumáticas mais comuns em idosos, sendo que o avanço da idade e o gênero influenciam no aumento da ocorrência nesta população (WU et al., 2019; ARMOND et al., 2014; TSUDA, 2017).

Os resultados encontrados nesta pesquisa condizem com outros estudos que indicam maior frequência de internações por fratura do fêmur entre as mulheres mais idosas (PORTO et al., 2019; OLIVEIRA e BORBA, 2017). Sobre as internações por fratura do fêmur com localização anatômica proximal, o perfil encontrado foi semelhante, corroborando com outras pesquisas (NETO et al., 2011; PAULA et al., 2015). Na sua grande maioria, este tipo de fratura requer internação para tratamento cirúrgico e apresenta maior risco de morbimortalidade e redução da qualidade de vida (PAULA et al., 2015; ALMEIDA et al., 2017).

Observa -se uma relação entre o aumento dos casos de fraturas do fêmur com o avanço da idade, sendo o gênero feminino o mais acometido (BORTOLON et al., 2011). Sabe-se que devido a deficiência de estrogênio, as mulheres na menopausa apresentam maior incidência de osteoporose, quando comparado com os homens. Assim, são mais suscetíveis as fraturas de quadril, coluna e punho (CHEN et al., 2018). O declínio da densidade óssea, a idade, histórico de fratura, tabagismo, etilismo e artrite reumatoide são os principais fatores de risco associados com as fraturas em mulheres pós-menopausa (WU et al., 2019; TSUDA, 2017). Análises mostraram que o status socioeconômico e baixo nível educacional também são fatores para a ocorrência da primeira fratura de estruturas do quadril, como o fêmur 
proximal (CHEN et al., 2018). Além disso, estes dados podem retratar a feminização do fenômeno de envelhecimento populacional (ARMOND et al., 2014). Isto se deve principalmente pelas diferenças encontradas na expectativa de vida entre os gêneros, sendo que na média geral, as mulheres vivem mais do que os homens (TRAVASSO et al., 2020).

As internações com desfecho de óbito por fratura do fêmur foram maiores entre as mulheres mais idosas, estando de acordo com alguns estudos epidemiológicos (BORTOLON et al., 2011; PORTO et al., 2019). Foi verificado uma associação entre indivíduos com idade mais avançada e o aumento da taxa de mortalidade por fratura do fêmur. Porém, alguns estudos mostram prevalência na mortalidade entre os homens mais idosos (PETERLE et al., 2019; OLIVEIRA e BORBA, 2017). A maior proporção de mulheres internadas por fratura do fêmur poderia justificar a prevalência dos óbitos hospitalares neste grupo. Porém, estudo realizado entre 2008 a 2018 sobre a mortalidade hospitalar entre as Regiões do Brasil, demonstrou maior mortalidade entre os homens internados. Observou-se ainda que os custos decorrentes deste agravo são altos e apresenta diferenças entre as Regiões brasileiras (PETERLE et al., 2019). As discordâncias entre os achados podem estar relacionadas com inúmeras variáveis decorrentes da geografia e clima, hábitos alimentares, ações em saúde, fatores de risco, constituição da população, e subnotificação dos casos (SOARES et al., 2014). Outros fatores de risco envolvidos podem ser a presença de mais comorbidades, alto risco cirúrgico em indivíduos nesta faixa etária, presença de deficiência cognitiva e gênero (EDELMUTH et al., 2018; NETO et al., 2011).

O fenômeno de envelhecimento pode aumentar a incidência de fraturas do fêmur se medidas preventivas não forem adotadas (BEER e GILES, 2005). Além de maior risco de mortalidade, usualmente após a ocorrência da fratura, os idosos apresentam algum nível de limitação física, comprometimento da saúde mental e redução da expectativa de vida (EDELMUTH et al., 2018; OLIVEIRA e BORBA, 2017; ALEXIOU et al., 2018). 
A distribuição da população idosa não é homogênea no Brasil, havendo diferenças regionais. A Região Norte apresenta a menor concentração de idosos, sendo 53,2\% representada por mulheres (TRAVASSO et al., 2020). Apesar disto, o envelhecimento populacional deve ser considerado como um dos grandes desafios para sistema público de saúde, que não está preparado para atender esta nova demanda. Estratégias como a educação em saúde e a prevenção das fraturas devem ser adotadas para reduzir os impactos negativos deste agravo. Além disso, o uso de dados secundários pode auxiliar no conhecimento das necessidades do Estado e servem de apoio nas estratégias voltadas para as demandas em saúde.

As principais limitações deste estudo estão associadas com o uso e a qualidade dos dados secundários disponibilizados no SIH/SUS, como a subestimação dos casos e a impossibilidade de se conhecer as causas que levaram às internações por fraturas do fêmur. Além disso, não foram utilizados dados populacionais, o que requer estudos futuros mais completos, principalmente devido as características demográficas do Estado de Roraima.

\section{CONSIDERAÇÕES FINAIS}

De acordo com a faixa etária e gênero, observou-se que as internações e os óbitos hospitalares por fratura do fêmur foram mais prevalentes nas mulheres octogenárias. Estratégias em saúde, como a prevenção de quedas, deve ser direcionada para a população idosa, em especial às mulheres, que são as mais acometidas por este agravo. Além disso, ações conjuntas que visam a melhoria do bem-estar e qualidade de vida devem ser adotadas para um envelhecimento mais ativo e saudável, considerando sempre a realidade e as demandas locais.

\section{REFERÊNCIAS}

ALEXIOU, K. I. et al. Quality of life and psychological consequences in elderly patients after a hip fracture: a review. Clin Interv Aging. v. 24, n. 13, p. 143-150, 2018. doi: $10.2147 /$ CIA.S150067 
ALMEIDA, D. O. et al. Internações por fraturas ósseas pelo SUS em idosos do município de Salvador (BA): um estudo descritivo do ano de 2015. Rev. Ciênc. Méd. Biol. v. 16, n. 3, p. 288-295, 2017. doi: 10.9771/cmbio. v16i3.24384

ARMOND, J. E. et al. Caracterização de idosos internados por queda acidental na cidade de São Paulo, 2013. Rev. Bras. Geriatr. Gerontol. v. 8, n. 4, p. 200-203, 2014.

BEER, C.; GILES, M. Hip fracture Challenges in prevention and management. Aus.Fam.Physician. v.4, n. 8, p. 673-676, 2005.

BORTOLON, P.C. et al. O perfil das internações do SUS para fratura osteoporótica de fêmur em idosos no Brasil: uma descrição do triênio 20062008. Cad. Saúde Pública. v. 27, n. 4, p. 733-742, 2011. doi: 10.1590/S0102$311 \times 2011000400012$

CARMO, W.M.; GOMES, L. S. M. Fraturas Trocantéricas. J Bone Joint Surg. v. 90, p. $700-7007,2008$.

CHEN, F. P. et al. Risk factors and quality of life for the occurrence of hip fracture in postmenopausal women. Biomed J. v. 41, n. 3, p. 202-208, 2018. doi: 10.1016/j.bj.2018.04.001

EDELMUTH, S. V. C. L. et al. Comorbidities, clinical intercurrences, and factors associated with mortality in elderly patients admitted for a hip fracture. Rev. bras. ortop. v. 53, n. 5, p. 543-551, 2018. doi10.1016/j.rboe.2018.07.014

MACEDO, G. G. et al. Fraturas do fêmur em idosos: um problema de saúde pública no Brasil. REAC. v. 6, n. 1112, p. 01-07, 2019. doi: 10.25248/reac. e1112.2019

NASCIMENTO, M.M. Queda em adultos idosos: considerações sobre a regulação do equilíbrio, estratégias posturais e exercício físico. Geriatr. Gerontol. Aging. v. 13, n. 2, p. 103-110, 2019. 
NETO, J. S. H. et al. Características epidemiológicas e causas da fratura do terço proximal do fêmur em idosos. Rev Bras Ortop. v. 46, n. 6, p. 660-667, 2011. doi: 10.1590/S0102-36162011000600007

OLIVEIRA, C. C.; BORBA, V.Z.C. Osteoporosis in brazilian patients awaiting knee arthroplasty. Acta Ortop Bras. v. 25, n. 4, p. 155-158, 2017. doi: 10.1590/1413-785220172504168827

PAULA, F. L. et al. Readmissão e óbito de idosos com alta após internação por fratura proximal de fêmur, ocorrida nos hospitais do Sistema Único de Saúde entre os anos de 2008 e 2010. Ver Bras Epidemiol. v. 18, n. 2, p. 439-453, 2015. doi: 10.1590/1980-5497201500020012

PETERLE, V.C. U. et al. Indicators of morbidity and mortality by femur fractures in older people: a decade-long study in brazilian hospitals. Acta Ortop Bras. v. 28, n. 3, p. 142-148, 2020. doi:10.1590/1413-7852202028032283

PORTO, A. O. et al. Características sociodemográficas e custo de hospitalizações por fratura de fêmur em idosos na Bahia. J Health NPEPS. v. 4, n. 2, p. 297-309, 2019. doi: 10.30681/2526101038233823

SANTOS, R. B. et al. Older adults' views on barriers and facilitators to participate in a multifactorial falls prevention program: Results from Prevquedas Brasil. Archives of Gerontology and Geriatrics. v. 92, p. 01-09, 2021. doi:10.1016/j.archger.2020.104287

SOARES, D. S. et al. Fraturas de fêmur em idosos no Brasil: análise espaçotemporal de 2008 a 2012. Cad. Saúde Pública. v. 30, n. 12, p. 2669-2678, 2014. doi: 10.1590/0102-311X00218113

TRAVASSO, G.F. et al. The elderly in Brazil: demographic transition, profile, and socioeconomic condition. Rev. bras. estud. popul. v.7, p. 01-27, 2020. doi: 10.20947/S0102-3098a0129 
TSUDA, T. Epidemiology of fragility fractures and fall prevention in the elderly: a systematic review of the literature. Curr Orthop Pract. v. 28, n. 6, p. 580-585, 2017. doi: $10.1097 / B C O .0000000000000563$

WU, S.C. et al. The influence of ageing on the incidence and site of trauma femoral fractures: across-sectional analysis. BMC Musculoskelet Disord. v. 20, p. 413-419, 2019. doi: 10.1186/s12891-019-2803-x

Enviado: Agosto, 2021.

Aprovado: Janeiro, 2022. 